\title{
The field epidemiology manual (FEM) wiki: a collaborative eLearning online portal to be launched at ESCAIDE
}

V Príkazský (vladimir.prikazsky@ecdc.europa.eu) ${ }^{1}$

1. European Centre for Disease Prevention and Control, Stockholm, Sweden

Citation style for this article:

Príkazský V. The field epidemiology manual (FEM) wiki: a collaborative eLearning online portal to be launched at ESCAIDE. Euro Surveill. 2010;15(44):pii=19701. Available online: http://www.eurosurveillance.org/ViewArticle.aspx?Articleld=19701

Article published on 4 November 2010

A field epidemiology manual (FEM) training resource, the FEM wiki project, has been developed to support the European Programme for Intervention Epidemiology Training (EPIET) and to serve as resource for any training in intervention epidemiology. The project (www. femwiki.com) is the result of a collaboration between a team of experts from the City ehealth Research Centre (CeRC) of the School of Community and Health Sciences (SC\&HS) in London and the European Centre for Disease Prevention and Control (ECDC), and will be formally launched and opened to the public at the European Scientific Conference on Applied Infectious Disease Epidemiology (ESCAIDE) on 11-13 November 2010 in Lisbon, Portugal.

The aim of the FEM wiki project is to make the training manual available online using a collaborative Web 2.0 platform that takes advantage of user-generated input while simultaneously certifying the scientific content through an editorial and review process. An editorial board consisting of field epidemiology experts has been established to convert the existing single document chapters, created by EPIET trainers, scientific coordinators and facilitators, into a set of hyperlinked wiki pages, each describing key epidemiological concepts. The training structure of the original chapters is preserved, and linked to a set of fora that support commenting and discussion.

The portal structure ensures the ECDC-recognised peerreviewed content, approved by the editorial board, is available alongside user-generated and organically expanding pages.

The aim is to gather resources and to offer a collaborative space for creation of training material with a diversity of formats and to provide a meeting point for opinions. The target audiences include the EPIET community; the wider field epidemiology training community, the European Public Health Microbiology Training Programme (EUPHEM) fellows and anyone working in disciplines related to epidemiology.
The vision for the portal is that it will serve as key resource for training delivered by ECDC and will attract a large online community of experts, expanding the content to establish it as the key online resource for epidemiologists around the world. 